\title{
COVID-19, Neuro-oncology and Safe Clinical Practice
}

\author{
Salman Sharif ${ }^{*}$ and Amanullah Kakar ${ }^{1}$ \\ ${ }^{1}$ Department of Neurosurgery, Liaquat National Hospital and Medical College, Karachi, Pakistan
}

The COVID-19 pandemic is a global catastrophe with grave medical, economic, and social outcomes creating a massive burden on healthcare systems worldwide. For the safety of the healthcare workers and to prevent cross-contamination among patients, the hospital management and clinicians are challenged to make drastic yet timely decisions for optimal management of patients seeking medical and surgical care from them.

The magnitude of the impact of this pandemic was underestimated mainly during the initial period of the pandemic, both in developed and developing countries. Due to the unpreparedness of healthcare systems and an exponential rise in cases, healthcare professionals were easy prey for the virus. This led to quarantine, isolation, and hospitalization of doctors of all specialties leading to a paucity of human resources, further limiting the provision of efficient healthcare. Protocols of essential services like neurosurgery had to be revised. Neurosurgeons all around the world had to halt or significantly reduce their elective surgeries [1]. Furthermore, all specialties had to develop a system of triage to prioritize healthcare delivery. For example, the World Federation of Neurosurgical Societies \& Pakistan Society of Neurosurgeons developed the following policies to limit the provision of essential neurosurgical services [2].

-Urgent Surgeries: They needed doing immediately; otherwise, it would have resulted in death or disability of the patient. This mostly included trauma (extradural hematoma, acute subdural hematoma, contusion, chronic subdural hematoma) acute intracranial hemorrhage with mass effect, shunt malfunction, myelomeningocele repair, spinal cord compression, and acute hydrocephalus).

-Early Surgeries: Cases that could be delayed for up to 2 weeks, which included newly diagnosed brain and spinal tumors.

-Elective Cases: Surgeries that can be performed 30 or more days later with no deleterious effects on the patient, which include, slow-growing tumors, prolapsed intervertebral disc, Chiari decompression, and tethered cord release.

\footnotetext{
*Corresponding Author: Salman Sharif, Department of Neurosurgery, Liaquat National Hospital and Medical College, Karachi, Pakistan; Email: sharifsalman73@gmail.com

Received: June 30, 2020; Revised: July 13, 2020; Accepted: July 21, 2020

DOI: https://doi.org/10.37184/lntbj.2708-7808.2.1
}

Managing neuro-oncology patients posed the greatest challenge. Since outpatient departments (OPD) were shut down in the majority of institutions except LNH during the strict lockdown, most patients with tumors presented with significant neurological symptoms through the emergency department (ER), whereas asymptomatic patients avoided or possibly delayed their routine OPD visits. This led to an insurmountable load on healthcare institutions that could not comply with the huge demand for personal protective equipment (PPE) due to a supply-demand mismatch. This imposed risk to healthcare professionals, which further limited the resources. These circumstances were further complicated by the non-availability of the COVID test in the initial phase of the pandemic.

Neuro-oncology represents a multidisciplinary field comprising of an oncologist, neurosurgeon, pathologist, radiologist, and radiation therapist. Postponing routine tumor board meetings resulted in a delay in the timely management of neuro-oncology patients. It was indeed a trying time for both surgeons and their patients, but the world authorities, including the Pakistan Society of Neurosurgeons, were quick to respond to provide comprehensive guidelines. These guidelines categorized tumors that needed urgent intervention and which could safely be postponed. With China successfully beating the COVID-19, they increased their production and exportation of PPE. The local availability of COVID-19 PCR in hospital settings let to a large number of cases being done rather than being postponed. COVID-19 patients were also successfully operated, as the hospital was now better able to manage these patients while simultaneously safeguarding the health of doctors and paramedical staff. The surgeons and staff felt more secure if they knew the COVID status of their patient, even if it was positive.

The impact of this pandemic was significant for all the neurosurgeons around the world. However, they were faced with varied circumstances. Due to the high morbidity and mortality of COVID-19 cases in Europe and America, it was often leading to the relocation of neurosurgical residents and consultants in COVID wards and ICU. This was the phase of the maximum distress among the neuro-oncological patients, as all resources were directed towards COVID patients. Similarly, our institution followed the general guidelines laid down by WFNS and PSN. Only emergency, life threatening cases were performed while all elective cases were deferred. 
Although we have yet to operate on a tumor patient who tested COVID +ve, we are fully prepared if we are faced with this situation. Multidisciplinary management of such patients were significantly facilitated by the widespread availability of online virtual meetings amongst various consultants.

One of the most significant concerns during this pandemic was the management of tumors that were approached through the Endoscopic transsphenoidal route. In their warning about the endonasal surgeries during the pandemic, the Stanford group suggested a standard protocol of essential PPE to reduce the risk [3]. However, with the widespread availability of safety equipment, these patients were operated after optimal preoperative preparation. This included at least 2 COVID-19 PCR to be negative performed 2 days apart, rather than a single negative report which sufficed for routine neurosurgical cases. This is now a part of the Pakistan Society of Neurosurgeons as well as International Neurosurgical societies guidelines $[2,4,5]$

The world at large is still not done with the calamity, though we are more alert and better prepared to face the situation. At one end, it is crucial to provide adequate facilities to safeguard the healthcare professional, and at the same time making quick decisions in the best interest of all patients. This especially includes oncology patients for whom time is a precious commodity. The time taken by the health system to gear up to neuro-oncology patients during this pandemic should be shortened while protecting our fellow health care workers.

\section{REFERENCES}

1. Bernhardt D, Wick W, Weiss SE, et al. Neuro-oncology management during the COVID-19 pandemic with a focus on WHO Grade III and IV Gliomas. Neuro Oncol 2020; 22(7): 928-35.

2. Pakistan society of neurosurgeons guidelines. Available at: https:// www.paksn.org/covid-19/.

3. Stanford recommendations in PPE. Available at: http://med. stanford.edu/neurosurgery.html.

4. AANS guidelines. Available at: https://www.cdc. gov/coronavirus/2019-ncov/hcp/testing-overview. html?CDC_AA_refVal=https $\% 3 \mathrm{~A} \% 2 \mathrm{~F} \% 2 \mathrm{Fwww} . \mathrm{cdc}$.

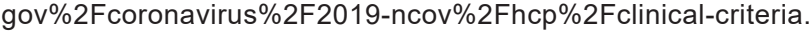
html.

5. Jean WC, Ironside NT, Sack KD, Felbaum DR, Syed HR. The impact of COVID-19 on neurosurgeons and the strategy for triaging non-emergent operations: a global neurosurgery study. Actaneurochirurgica 2020; 162: 1229-40. 